\title{
Effectiveness of fully documented fisheries to estimate discards in a participatory research scheme
}

Mortensen, Lars O.; Ulrich, Clara; Olesen, Hans Jakob; Bergsson, Heidrikur ; Berg, Casper Willestofte; Tzamouranis, Nikolaos; Dalskov, Jørgen

Published in:

Fisheries Research

Link to article, DOI:

10.1016/j.fishres.2016.11.010

Publication date:

2017

Document Version

Publisher's PDF, also known as Version of record

Link back to DTU Orbit

Citation $(A P A)$ :

Mortensen, L. O., Ulrich, C., Olesen, H. J., Bergsson, H., Berg, C. W., Tzamouranis, N., \& Dalskov, J. (2017). Effectiveness of fully documented fisheries to estimate discards in a participatory research scheme. Fisheries Research, 187, 150-157. https://doi.org/10.1016/j.fishres.2016.11.010

\section{General rights}

Copyright and moral rights for the publications made accessible in the public portal are retained by the authors and/or other copyright owners and it is a condition of accessing publications that users recognise and abide by the legal requirements associated with these rights.

- Users may download and print one copy of any publication from the public portal for the purpose of private study or research.

- You may not further distribute the material or use it for any profit-making activity or commercial gain

- You may freely distribute the URL identifying the publication in the public portal 
Full length article

\title{
Effectiveness of fully documented fisheries to estimate discards in a participatory research scheme
}

\author{
Lars O. Mortensen $^{\mathrm{a}, *}$, Clara Ulrich ${ }^{\mathrm{a}}$, Hans Jakob Olesen ${ }^{\mathrm{a}}$, Heiðrikur Bergsson ${ }^{\mathrm{b}}$, \\ Casper W. Berg a , Nikolaos Tzamouranis a , Jørgen Dalskov ${ }^{a}$ \\ a Institute for Aquatic Resources, Technical University of Denmark, Denmark \\ ${ }^{\mathrm{b}}$ Ministry of Food, Agriculture and Fisheries of Denmark, The Danish AgriFish Agency, Copenhagen, Denmark
}

\section{A R T I C L E I N F O}

\section{Article history:}

Received 6 September 2016

Received in revised form

18 November 2016

Accepted 19 November 2016

Handled by A.E. Punt

\section{Keywords:}

CCTV

Video inspection

Landing obligation

FDF

Electronic monitoring

Precision

\begin{abstract}
A B S T R A C T
A key challenge for fisheries science and management is the access to reliable and verifiable catch data. In science, the challenge is to collect reliable, precise and traceable data to provide sound advice. In management, the challenge is that catch documentation is necessary to enforce regulations. Currently, catch inspection at sea, self-reporting through e-log and on-board observers are the primary methods to document catches at sea. However, at-sea control and on-board observers are costly and have limited coverage, while self-reporting is susceptible to fraud and provides limited coverage. New cost-effective methods are currently emerging involving Remote Electronic Monitoring (REM) and on-board cameras. Previous studies have tested REM with promising results. However, evaluation of the potential biases of REM is needed before full benefits can be obtained. We deployed REM with on-board cameras on 14 fishing vessels and were able to inspect 56\% of 1523 hauls made in the 6 month trial period, using an estimated 582 man-hours of video audit. The results showed an overall good agreement between the fishers self-reported discards and the video inspectors discard estimates. However, there was large variation in precision between individual vessels and species. Additionally, trial setup and process errors were shown to have a large effect on the precision of the video inspectors discard estimates. Nevertheless, despite challenges, REM was evaluated to have the potential to streamline monitoring and scientific documentation in a medium-size fishing fleet.
\end{abstract}

(c) 2016 Elsevier B.V. All rights reserved.

\section{Introduction}

In fisheries science, experiments are conducted on scientific vessels and/or using scientific observers on board commercial fisheries vessels. Both methods are costly, resulting in limitation in data collection that can challenge the reliability of quantitative estimates derived from the experiments. Additionally, monitoring the compliance with the Landing Obligation (LO) from the 2013 reform of the Common Fisheries Policy (CFP) (EU, 2013), to be implemented between 2015 and 2019, will require managers to ensure that all catches are documented. Finding ways of ensuring cost-effective high quality catch data is therefore a challenge for both the science and management.

Remote Electronic Monitoring (REM) has been suggested as a cost-effective solution to document the on-board activities on fishing vessels and to fully document catches (e.g. Dalskov and

\footnotetext{
* Corresponding author.

E-mail address: laomo@aqua.dtu.dk (L.O. Mortensen).
}

Kindt-Larsen, 2009; Kindt-Larsen et al., 2011; Mangi et al., 2015; Marine Management Organisation, 2013; McElderry et al., 2003; Needle et al., 2015; Ulrich et al., 2015; van Helmond et al., 2015). REM is a full documentation tool that uses closed-circuit-television (CCTV) cameras, GPS and sensors to monitor the fishing operations in-situ. Results from the REM can be used to verify logbook reports from the fishers, suggesting that REM can be used to scientifically verify participatory monitoring programs in the fisheries and as a control tool in fisheries management. However, it is necessary that REM has the sufficient precision and accuracy to verify the fishing activities.

Many previous studies have evaluated REM to be largely reliable and accurate (Ames et al., 2007; Kindt-Larsen et al., 2011; Needle et al., 2015; Stanley et al., 2009, 2011; Ulrich et al., 2015; van Helmond et al., 2015). However, most previous studies included fisheries (such as hook and line fisheries) where it was easy to identify individual fish in the catch (Ames et al., 2007; McElderry et al., 2003) or only focused on a single species in a mixed fishery, usually cod (Gadus morhua) (Kindt-Larsen et al., 2011; van Helmond et al., 2015). Within the EU, only the Scottish REM trials have focused 
on monitoring the discard of multiple species in a mixed demersal trawl fishery (Needle et al., 2015), along with unpublished trials at the Danish AgriFish Agency (HeiĐrikur Bergsson pers. com). We use the data collected during a Danish fisheries trial (Mortensen et al., 2016) to expand on the current understanding on the applicability of REM in mixed fisheries. We aim to evaluate the discard estimates made by REM video inspectors for several species and contrast the estimates with the reports of fishers and on-board observers to estimate precision and accuracy of the REM observations.

\section{Materials and methods}

Data for this study were obtained from the Danish project MINIDISC conducted in Danish and Norwegian waters (Mortensen et al., 2016). The aim of MINIDISC was to evaluate the effect of free gear selection in a catch quota management scheme and included 12 demersal trawlers and 2 Danish seiners. REM was used as a support tool to ensure compliance with the trial guidelines. Each vessel reported catches and discards on a haul by haul basis for seven commercial species, for the period from September 17th 2014 to July 15th 2015. The commercial species included were: cod, hake (Merluccius merluccius), haddock (Melanogrammus aeglefinus), whiting (Merlangius merlangus), saithe (Pollachius virens), plaice (Pleuronectes platessa) and Norway lobster (Nephrops norvegicus).

Catch and discard data were logged by the fishers on a haul-byhaul basis, either through the national eLog system administrated by the Danish AgriFish Agency or through the software interface of the REM system on-board the vessel. All vessels were equipped with REM, including cameras, GPS and gear sensors, all connected to a control box where data were stored on two mirrored harddiscs. Video recording started when the vessel left the harbour and automatically turned off when entering the harbour again, using the GPS signal to trace position. Cameras recorded activity on the trawl deck and in the catch-handling area, with at least one camera focused directly on the discard chute and the end of the conveyer belt, to the extent permitted by the layout of the handling area. Data from the REM were directly uploaded to local servers using the $4 \mathrm{G}$ network when vessels were within coverage, and consisted of video coverage of the entire fishing operation, vessel position and timing of gear deployment and retrieval. Fishers were instructed to sort all discard of the seven species into baskets and show the contents to the camera before discarding.

The REM system and software used to analyse video material was developed by Anchor lab K/S (www.Anchorlab.dk), with a unit price of 45.000 DKK for a system ( 1 DKK/0.14 USD, 14th November 2016). REM systems were installed on the vessels by a company specialised in ship electronics (SkibsElektro), with the installation cost of approximately 30,000 DKK per vessel, depending on the home harbour and size of the vessel. Due to the wireless transmission of data, operational cost of the REM were relatively low (roughly $10,000 \mathrm{DKK}$ in total, all trips and vessels included) compared to other trials using REM technology that require changing hard disks at regular intervals, which have an annual running cost per vessel of approx. 30,000 DKK (van Helmond et al., 2015). The difference in cost is likely due to man hours spent changing hard disks, which are absent in wireless transmission.

A team of six video inspectors verified the discard, as data were uploaded to the servers. Video inspectors were University students with a background in fisheries science, but with no prior video inspection training. Introductory meetings with the video inspectors were held before initiation of inspection, where the inspectors were instructed in the video inspection procedure and the first week of inspection was done in pairs, to obtain a common understanding of how the inspection should be conducted.
Inspectors would access the oldest trip from participating vessels, which had not been inspected. Hauls would be identified, using data from pressure sensors on wire winches, which indicate deployment and retraction of gears. Inspectors would note time of deployment and subsequently fast-forward to gear retraction and note the time. The sorting and discarding process would then be inspected at $2 \mathrm{x}-5 \mathrm{x}$ times speed, with pauses when fishers would display discard buckets to the camera.

Fishers were instructed to sort all discard of the selected species into discard-baskets and show the basket to the camera before discarding. Discard was verified, species identified and discard amounts were estimated by inspectors, along with the monitoring of fish being discarded without sorting. Discard weight was estimated by inspectors by counting baskets. Partly full baskets were estimated in percentages. Subsequently, the number of baskets was multiplied by $35 \mathrm{~kg} /$ basket. The weight of $35 \mathrm{~kg} /$ basket was used as best estimate of the weight of a basket with mixed fish in the discard size class, derived from experiences in the Danish Agrifish Agency and research on the research vessel DANA. Inspectors did not know the self-reported catch composition, catch amounts or discard amounts, from the fisher, in advance.

To obtain "true" discard values, some trips were made with an on-board observer, who measured all discard of each species, including length and weight. The final dataset thus comprised of discard estimates from fishers, video inspectors and on-board observers.

Video inspection did not start until the trial was half way through, which also meant that video errors and positioning could not be corrected when they were detected. Instead, video inspectors commented on each video in terms of challenges, when inspecting the video and possible errors. To optimise the data set, these errors and challenges were categorised into three groups: camera errors, protocol errors and inspection errors. Camera errors could be hauls with missing footage, reduced video quality and other physical challenges with the video stream. Protocol errors were situations where the fishers did not behave according to experiment agreements and either forgot to show the discard basket to the camera, or did not use a discard basket, etc. Inspection errors were hauls where camera coverage was not sufficient and either discard buckets were out of view of the camera or cameras did not cover the sorting table.

Only hauls with registered discard from either fisher or video inspector were included in the analysis, to avoid a zero inflated data set, due to several trips where both fisher and video inspector agrees on zero discard. All statistical analysis was done in the $\mathrm{R}$ statistical software (Full of Ingredients, version 3.2.0) (R Core Team, 2014).

We quantified systematic differences in estimation between fishers and video inspectors. A systematic difference is the continuous over- or underestimation of discard by one method (e.g., fishers discard estimate) in relation to the opposing method (e.g., video inspectors discard estimate). The systematic difference between fishers and video inspectors' ability to estimate discard weight was analysed using normal linear regression. The linear regressions were conducted with video inspectors' discard estimates as the dependent variable and fishers' estimates as explanatory variable, using the $\mathrm{lm}$ function in R-package base. The intercept was set to 0 , which assumes that agreeing on zero discard would be easy. This also allows easy interpretation of the model fit, because the systematic difference can be inferred from the estimated slope of the regression line, $\alpha$. The model may thus be written as:

$y_{i}=\alpha x_{i}+\varepsilon_{i}$

where $y$ is the video inspectors' estimates of discard in $\mathrm{kg}$ and $x$ is the fishers' estimates of discard in $\mathrm{kg}$ and $i$ is an index for trip. 
Although the data were not normal distributed, with no simple transformation available to correct this, normality of $\varepsilon$ was assumed by the model nevertheless. For this reason, confidence intervals were computed using ordinary bootstrapping $(n=400)$, since this method does not assume normality. Initially, all data were analysed together for systematic differences in discard estimation, across all vessels and species, with a subsequent differentiation between vessels and species.

To analyse the effect of errors on a potential systematic difference in discard estimation, all regressions were completed on subsets of the overall data set, either removing all hauls with identified challenges, or only removing selected errors. This enabled the assessment of the effects of different errors on the discard estimation.

\section{Results}

Only video material from 12 of the 14 vessels participating in the trials was inspected by the video inspectors, due to complications in the catch registration of the remaining two vessels. A total of $13,514 \mathrm{~h}$ of at-sea were recorded during the MINIDISC project. From GPS and pressure/weight sensors, it was estimated that the vessels were steaming around $51 \%$ of the time during fishing operations, resulting in approximately $6622 \mathrm{~h}$ of trawling. As the project was time-constrained within a given period and the staff for video inspection was limited, we were unable to inspect all hauls. Nevertheless a total of $3723 \mathrm{~h}$ could be inspected by the video inspectors, covering 853 hauls across 10 demersal trawlers (Table 1). Thus,
Table 1

Vessels sizes and areas.

\begin{tabular}{lll}
\hline Vessels & Area & Size $(\mathrm{m})$ \\
\hline A & Skagerrak & 16 \\
B & Skagerrak & 18 \\
C & North Sea & 31 \\
D & North Sea & 25 \\
E & North Sea & 31 \\
F & Baltic Sea & 16 \\
G & Baltic Sea & 12 \\
H & Baltic Sea & 17 \\
I & North Sea & 29 \\
J & Skagerrak & 16 \\
K & Skagerrak & 18 \\
L & Skagerrak & 17 \\
\hline
\end{tabular}

$56 \%$ of the 1523 hauls were inspected by video inspectors, using on average $41 \mathrm{~min}$ per haul. Hauls were not randomly selected, but taken in chronological order as they entered the data stream. Of the inspected hauls, 379 hauls had attached comments from the video inspectors: 72 camera comments, 238 protocol comments and 76 inspection comments. Thus, alterations in protocol were the largest challenge for the video inspectors.

The initial regression across all data, showed a systematic underestimation of discard from the video inspectors in relation to the fishers (Fig. 1) $\left(\alpha=0.68, \mathrm{R}^{2}=0.60, \mathrm{CI}=0.57-0.84\right)$, indicating an average discard estimate from the video inspectors of $0.68 \mathrm{~kg}$ per $\mathrm{kg}$ discard estimate from fishers. The median deviation in discards estimates between video inspectors and fishers (Table 2) was between 2 and $13.5 \mathrm{~kg}$ for each species across all hauls. However,
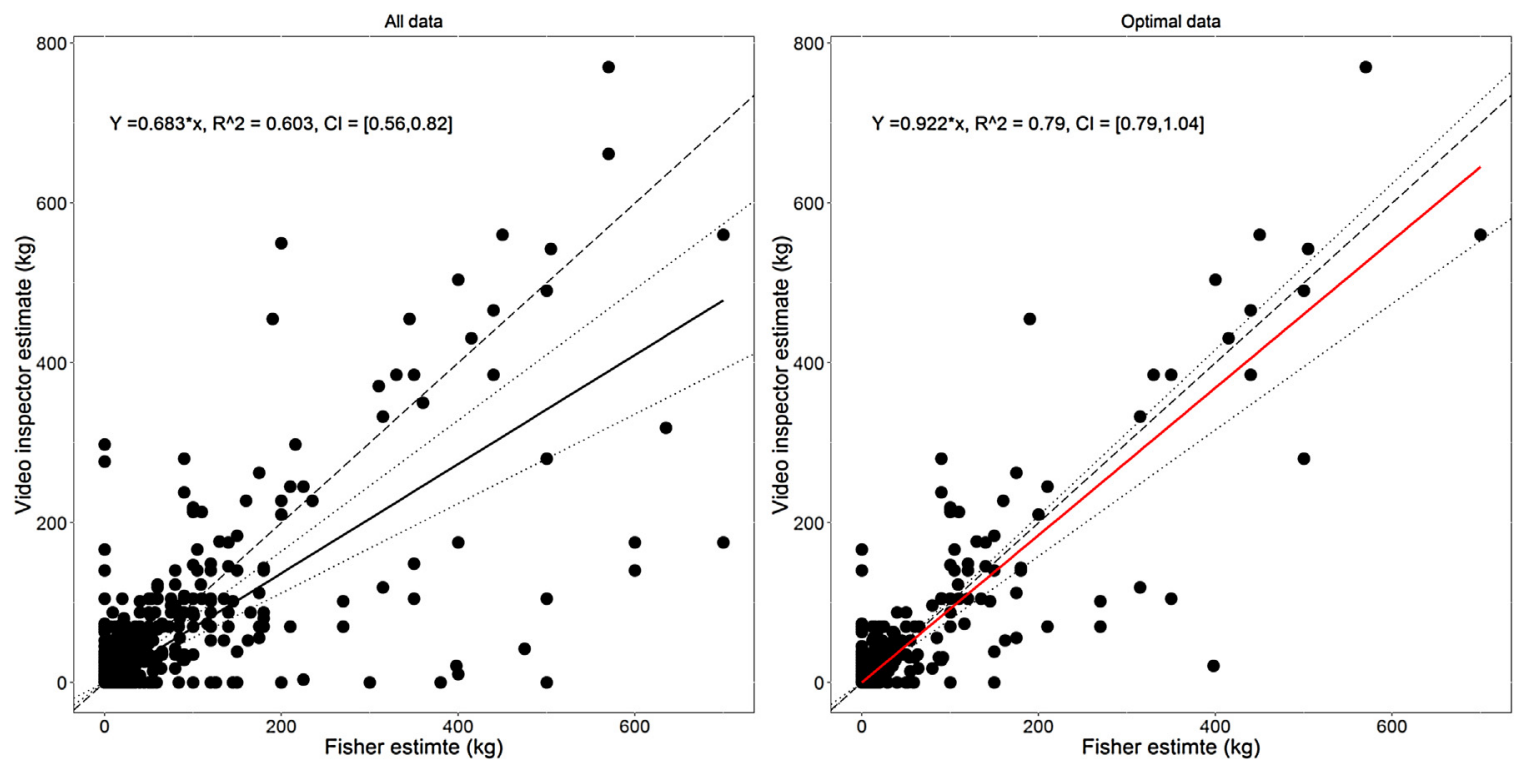

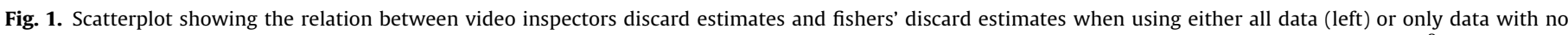

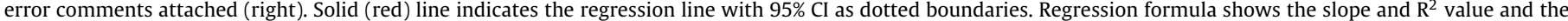

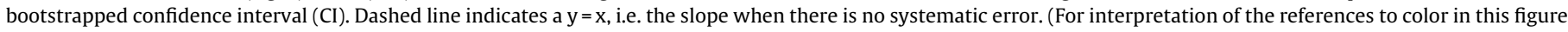
legend, the reader is referred to the web version of this article.)

Table 2

Overview of median discard estimates from video inspectors and fishers during the trial, along with 10 percentile and 90 percentile.

\begin{tabular}{|c|c|c|c|c|}
\hline Species & $\mathrm{n}$ & Video inspectors & Fishers & Difference \\
\hline Cod (Gadus morhua) & 777 & $21[0,105]$ & $9[0,100]$ & $10.5[0,66]$ \\
\hline Haddock(Melanogrammus aeglefinus) & 190 & $0[0,18]$ & $4,[1,15]$ & $4.0[0,16]$ \\
\hline Hake (Merluccius merluccius) & 25 & $0[0,33]$ & $2[0,9]$ & $2.0[0,27]$ \\
\hline Nephrops (Nephrops norvegicus) & 86 & $0[0,24]$ & $7[2,25]$ & $7.0[1,28]$ \\
\hline Plaice (Pleuronectes platessa) & 347 & $14[0,64]$ & $4[0,50]$ & $13.5[0,67]$ \\
\hline Saithe (Pollachius virens) & 35 & $0[0,4]$ & $2[1,9]$ & $2.0[1,10]$ \\
\hline Whiting (Merlangius merlangus) & 52 & $0[0,33]$ & $2[0,23]$ & $3.0[0,16]$ \\
\hline
\end{tabular}



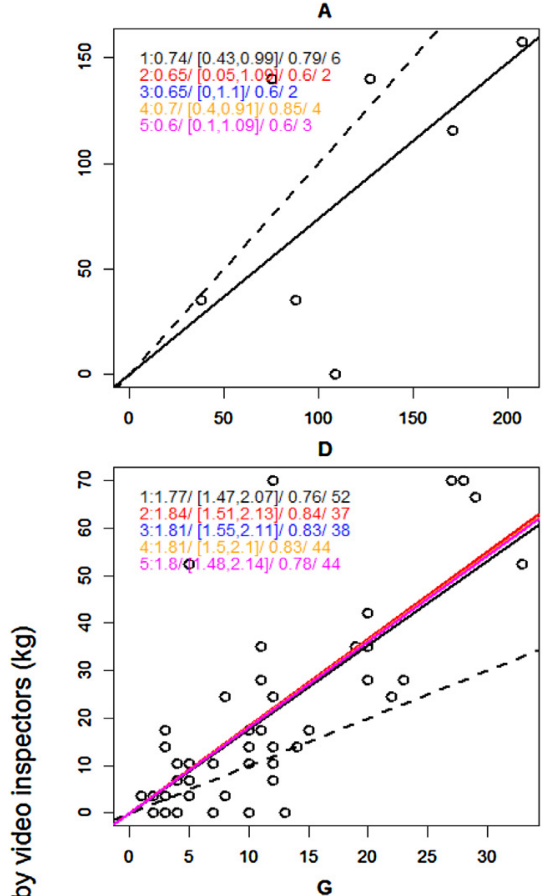
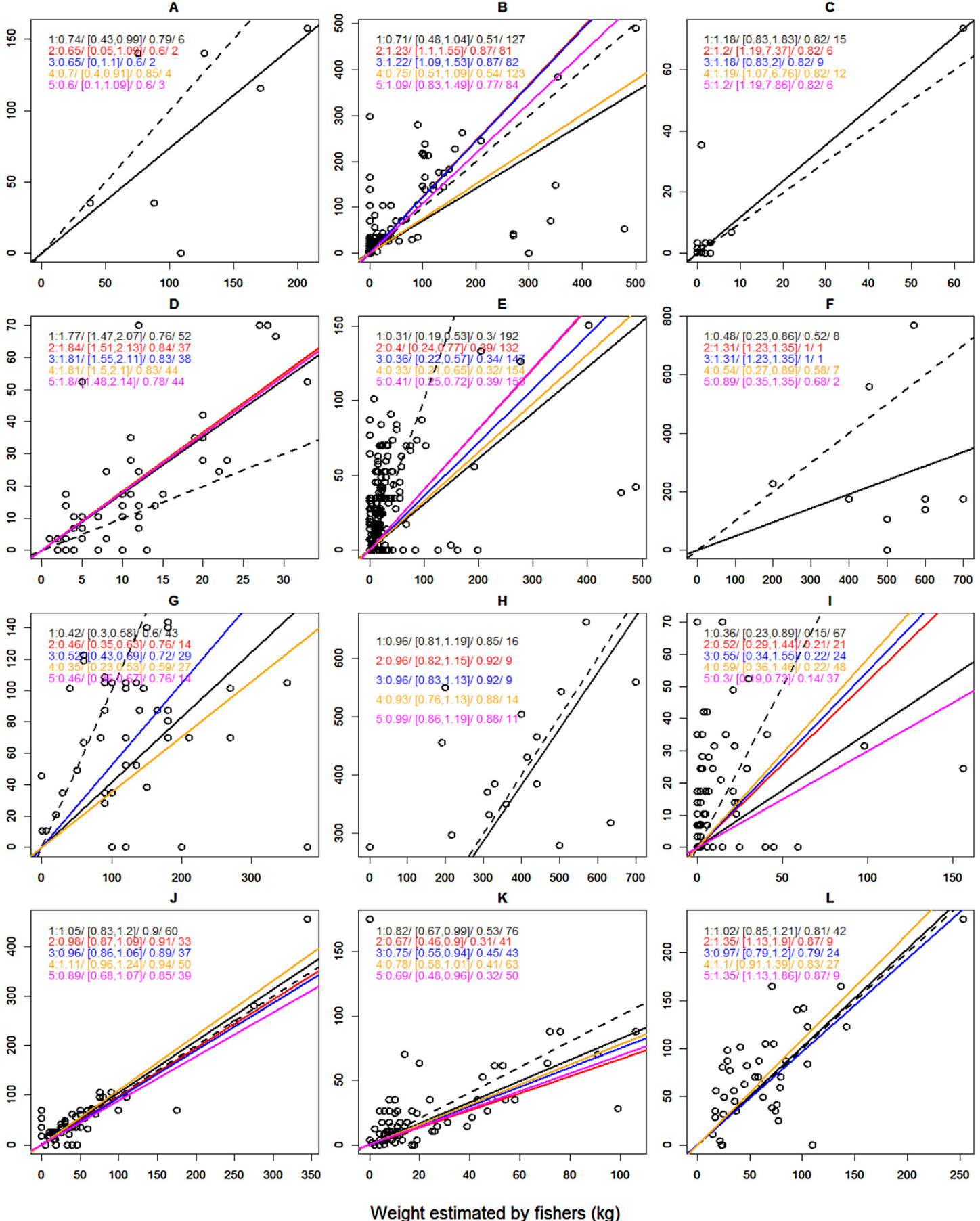

Weight estimated by fishers $(\mathrm{kg})$

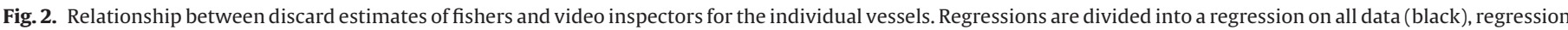

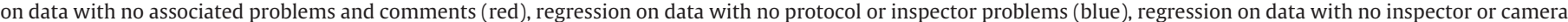

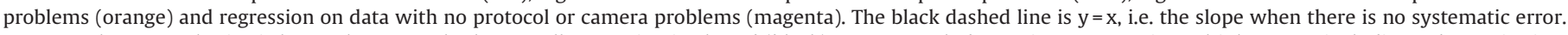

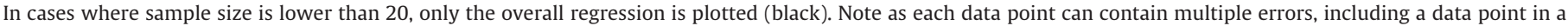

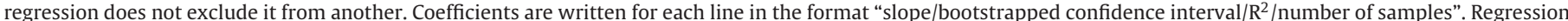

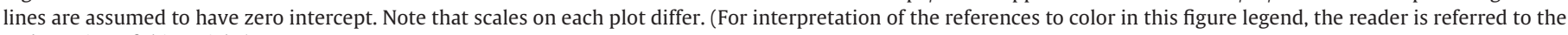
web version of this article.)

removing hauls with comments from video inspectors that may have been biased towards errors, improved the fit (Fig. 1) ( $\alpha=0.92$, $\left.\mathrm{R}^{2}=0.79, \mathrm{CI}=0.78-1.05\right)$, narrowed the bootstrapped confidence interval and also removed the systematic underestimation, yielding an overall agreement of discard estimates between fishers and video inspectors.

However, vessel differences are likely to occur, depending on vessel layout and crew. Agreement between fishers and video inspectors were thus analysed for each vessel (Fig. 2). Sample size was low on vessels $\mathrm{A}, \mathrm{C}, \mathrm{F}$, and $\mathrm{H}$, and systematic differences detected in these vessels are therefore not likely to be representative, as one extra data point could change the systematic difference. Using all data showed a varying result in agreement between video inspectors and individual fishers. The systematic difference in discard estimates were lower than $20 \%$ on vessels J, K, and L, while it was higher than $50 \%$ on vessels $\mathrm{E}, \mathrm{G}$, and $\mathrm{I}$. 

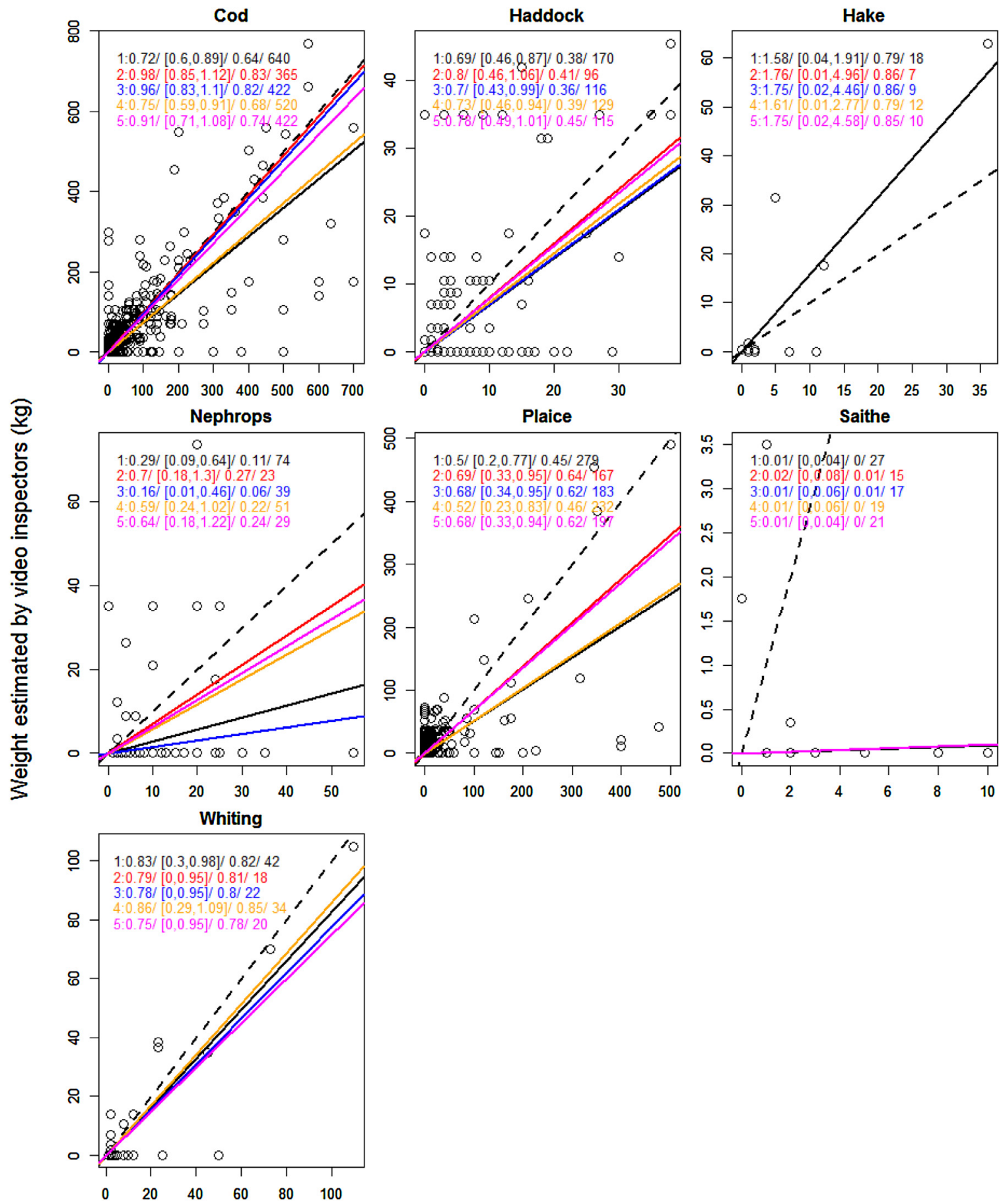

Weight estimated by fishers $(\mathrm{kg})$

Fig. 3. Relationship between discard estimates of fishers and video inspectors, with regression lines divided by species. Regressions are divided into a regression on all data (black), regression on data with no associated problems and comments (red), regression on data with no protocol or inspector problems (blue), regression on data with no inspector or camera problems (orange) and regression on data with no protocol or camera problems (magenta). In cases where sample size is lower than 20 , only the overall regression is plotted (black). Note as each data point can contain multiple errors, including a data point in a regression does not exclude it from another. Coefficients are written for each line in the format "slope/bootstrapped confidence interval $/ \mathrm{R}^{2} /$ number of samples". Regression lines are assumed to have zero intercept. Note that scales on each plot differ. (For interpretation of the references to color in this figure legend, the reader is referred to the web version of this article.)

Only three vessels (D, J, L), with a sufficient data set, had a $\mathrm{R}^{2}$ higher than 0.75 . However the removal of all hauls with an attached comment (indicating a potential bias in the data) increased the number of vessels with an $R^{2}$ higher than 0.75 to five $(B, D, G, J$, $\mathrm{L})$. This indicates that video-system errors can explain some of the systematic difference on the individual vessels. Using the spread of the regression lines on Fig. 2 within each vessel enabled an analysis os the effect of the different error categories on the agreement of discard estimates. Video errors did not have any overall effect on the discard estimates for the vessels D, J, and K, as indicated by almost identical regression slopes. Protocol errors had an effect on the agreement in discard estimates on vessels B and G, resulting in 
video inspectors underestimating discards in relation to the fishers. Protocol errors for vessels $\mathrm{E}$ and $\mathrm{L}$ also resulted in video inspectors underestimating discards, with camera errors adding to this effect. Lastly disagreement on discard estimates for vessel I were exacerbated by observer errors. Thus, despite the overall good model fit and agreement in discard estimates when removing data, the systematic difference within most vessels was still large.

Analysing discard estimates by species showed that hake and saithe were discarded only few times ( $<15$ times), which was also reflected by a poor fit between fishers and video inspectors estimates (Fig. 3). For cod, the removal of all video observations with errors resulted in a good agreement of discard estimates between fishers and video inspectors, with almost no systematic error. However, the remaining species (haddock, Nephrops, plaice and whiting) exhibited a considerable systematic error in the bootstrapped confidence intervals, even with all problematic hauls removed (Fig. 3).

The video inspector discard estimates were also compared to objective "true" values, by conducting fishing trips with onboard observers from 3 trips and 11 hauls. Comparisons between fishers' discard estimates and on-board observers were not conducted as fishers would have access to the discard estimates of the observers and could thus be biased in reporting their own discards. The analysis showed an overall good agreement between video inspectors and on-board observers (Fig. 4) $\left(\alpha=0.91, \mathrm{R}^{2}=0.99\right.$, $\mathrm{CI}=0.68-1.16)$. However, the bootstrapped confidence interval was relatively large, which could be due to the analysis being driven by one large discard estimate $(>400 \mathrm{~kg}$ ). However, dropping the this discard estimate did not change the fit significantly $\left(\alpha=0.90, \mathrm{R}^{2}=0.76, \mathrm{CI}=0.67-1.23\right)$. Removing the large discard estimate and disaggregating the analysis at species level showed a larger disagreement between the on-board observers and the video inspectors (Fig. 5). Cod estimates were in generally good agreement,

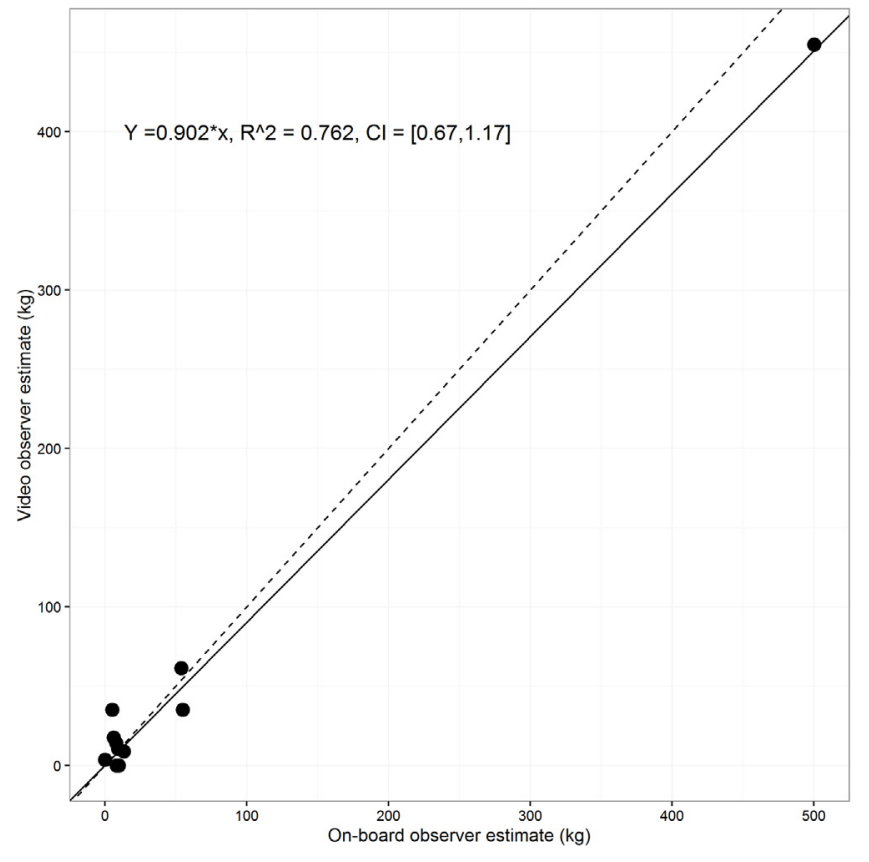

Fig. 4. Scatterplot showing the correlation between the average discard estimate per haul for video inspectors and on-board observers. The dotted black line shows the isocline with a 1:1 ratio between estimates and solid black line shows best model fit.

but the model fit was poor $\left(\alpha=0.94, \mathrm{R}^{2}=0.18, \mathrm{CI}=0.40-2.1\right)$. Haddock, hake, Nephrops and saithe were not sampled often enough to provide a model, while plaice $\left(\alpha=0.59, \mathrm{R}^{2}=0.56, \mathrm{CI}=0-1.24\right)$

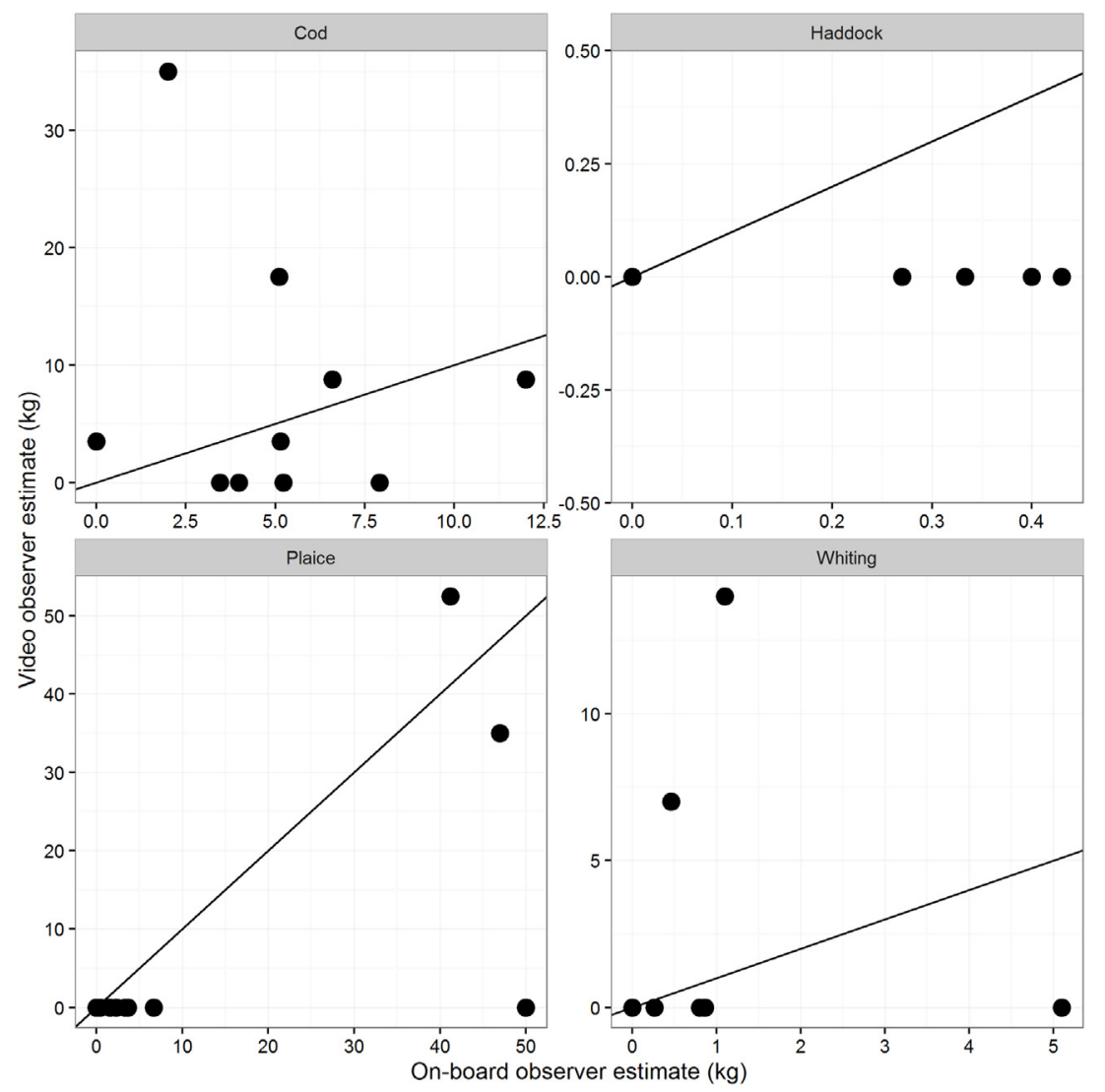

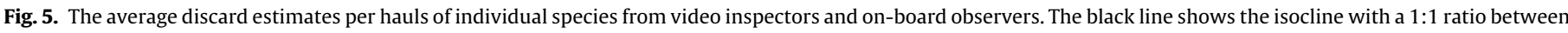
estimates. Only cod, haddock, plaice and whiting were caught on the trips with on-board observers. 


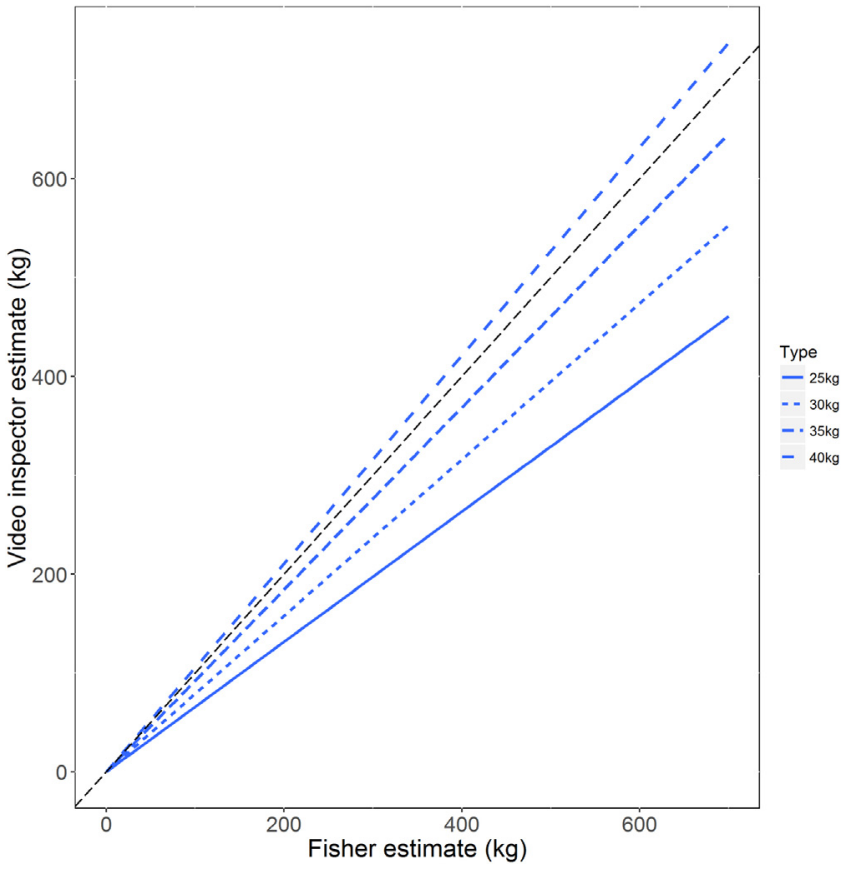

Fig. 6. Effect of changing weight of discard buckets. Regression lines indicate regressions on video inspectors discard estimates of number of buckets multiplied by weights. Thin dashed line is an isocline with the intercept of 0 and slope of 1 , indicating the perfect relation between fishers and video inspectors.

and whiting $\left(\alpha=0.64, \mathrm{R}^{2}=0.05, \mathrm{CI}=0-11\right)$ were generally underestimated, but with a poor model fit as well. Thus, only on an overall level did video inspectors and on-board observers agree on the discard amounts, which seemed to be driven by good cod estimates.

Lastly, to analyse the effect of varying "true" baskets weights, linear regression between fishers discard estimates and video inspectors discard estimates was conducted, while varying the basket weight on the video inspectors discard estimate to $25 \mathrm{~kg}, 30 \mathrm{~kg}$, $35 \mathrm{~kg}$ and $40 \mathrm{~kg}$ (Fig. 6). The results showed that video inspectors would underestimate the fishers discard estimate by approx. 33\% when using $25 \mathrm{~kg}$ per basket, while a slight overestimate would occur for $40 \mathrm{~kg}$ baskets.

\section{Discussion}

We estimated the accuracy of discard estimates for multiple species using electronic monitoring (EM) and CCTV surveillance. The results showed that, on an overall fleet level, there was a tendency for video inspectors to underestimate discards by $32 \%$ compared to fishers' discard estimates This supports the findings of Ulrich et al. (2015) and van Helmond et al. (2015), which suggest that, despite offering a promising way to supplement existing on-board observer control, the REM approach applied in this trial needs some adjustments to optimise video inspector accuracy. This is emphasised by the results obtained when removing all observations where video inspectors commented on the poor quality of the REM data, which showed an overall agreement between fishers and video inspectors on the discard estimates, thus supporting the findings of Stanley et al. (2011) and Kindt-Larsen et al. (2011). The high accuracy was also underpinned by the comparison between video inspectors and on-board observers, where there was a good overall agreement between the discards amounts in each haul. Fast inspection of the video quality and protocol compliance thus seems essential to ensure data are of sufficient quality for accurate video inspection.
However, resolving the discard estimates to a species level, showed distinct differences in discard estimates between video inspectors and fishers/on-board observers. All species, except cod, were not estimated properly by the video inspectors compared to both the fisher's discard estimates and to the on-board observer's discard estimates. This can be due to the challenge of identifying species on the cameras, when discards are placed in baskets (video inspectors pers. com). On the other hand, cod discard estimates from video inspectors were in good agreement with both fishers and on-board observers. Cod was the main discarded species, and it could be possible that the high level of agreement result from an increased likelihood of guessing the right species, compared to the other species. Additionally, cod was discarded in larger numbers than the other species, which means that few kilograms difference between estimates have less effect on the comparison. The discrepancy in discard estimates at the species level could also be derived from a lack of training of the video inspectors. Identifying species on a screen is not comparable to identifying species in the laboratory. While the time did not permit this in the current study, formal training as conducted in Needle et al. (2015), could have improved the results in the current trial significantly. Analysing the discard estimates on individual vessels also provided a mixed picture of the REM as monitoring tool. Of the 12 evaluated vessels, 4 had a sample size too low to provide any reliable accuracy estimates, which underpin the importance of maintaining a high control frequency. However there were no correlation between the number of hauls inspected by video inspectors and the agreement between fishers and video inspectors.

Thus, while on-board observers provided the least biased discard estimates, the most high quality discard estimates from the video inspectors can provide estimates with some degree of precision. Balancing precision against costs and degree of coverage, it may still be argued that video inspectors and REM can provide useful discard estimates on a haul-by-haul basis in a more cost-efficient approach than on-board observers, as the price of a haul inspected by REM opposed to on-board observers are substantially lower and can, thus provide a wider coverage, increasing the fleetwide discard estimate. From the current trial, the overall expense on REM was 1 mill. DKK, including 75,000 DKK for systems and installation, along with 100,000 DKK in salary for student helpers. Comparably, the price for observing 1523 hauls using an on-board observer would be 3 mill, assuming aproximately 3 hauls per day. Thus, using REM decreased the expense by $66 \%$.

\subsection{Errors affecting analysis}

Video inspectors reported on errors in the video observation process, which was categorised into three groups: camera errors, protocol errors and inspection errors. Analysing the effect of the different error types on the agreement in discard estimates showed that the protocol issues of the fisher were the largest challenge for the video inspectors' ability to estimate discard. Protocol issues could be fishers blocking the view of the discard, fishers discarding fish before it was demonstrated to the camera, etc. On the overall discard estimate agreement, it was seen that protocol issues accounted for the largest part of the errors and removing the hauls with issues increased the overall agreement substantially. On a vessel level, four vessels repeatedly demonstrated protocol errors, which resulted in an underestimation of discards by the video inspectors. Protocol issues likely arise due to the static position of the cameras, which means that video inspectors cannot change the camera angle to obtain a better view of the discard. Camera errors also accounted for a large part of the errors. However, the camera errors did not seem to affect the agreement of discard estimates to any extent. It is likely that hauls with defected or dirty cameras were not analysed by the video inspectors and thus, represent 
uninspected hauls, rather than hauls with incorrect discard estimates. Lastly, inspection errors only affected the discard estimate agreement on one vessel. However, no obvious reason could explain this effect as the vessel (I) had an adequate camera setup, except that all cameras pointed to the sorting belt, thus providing little room in the picture to see the discard baskets.

Thus, while the overall trial showed that REM could provide reasonable discard estimates on a fleet and catch basis, the analysis performed at the scale of individual vessels and species provided weaker agreement in the discard estimates. To improve discard estimates from video inspectors, protocols and technical applications needs to improve. In our trial we noted a number of caveats, which could be improved in future trials.

First of all, the sorting of discards into baskets for discard estimation was identified as a major issue. The work process added extra time and burden to the existing work of sorting and rinsing catches and also made it hard to identify species for the video inspector. Also, the applied average weight of a basket is likely not to fit the actual weight as basket weight varies with fish size, structure and if the basket is topped with fish. This is supported by Fig. 6, which displays the variation in discard estimates depending on the applied average basket weight. Using baskets also introduces the risk of having a mix of fish in the bottom, which cannot be identified. To improve on the visibility of discards and increase the chance for accurate species identification, it is likely that discard should be identified directly on the sorting belt, just before the discard chute. This would allow ease species identification and length measurements. Additionally, to improve protocols, fishers should be instructed not to leave fish in piles on the band, but to smooth out the layer. Currently the Danish AgriFish Agency is conducting trials with this method, and the first outcomes are promising (Heidrikur Bergsson, unpublished data).

Second, the trial also found that camera positioning was important for the image quality and ease of discard estimates. Camera positions over the sorting belt provided good overview of the sorting process. However, these cameras had a tendency to collect moisture and because the camera houses are dome-shaped and pointing straight down, the drip collection point would be with in the field of view, thus obscuring vision of the video inspectors. Changing the mounting position to allow for tilting the camera, and thereby changing the drip collection point away from the focus point of the camera, would solve this issue. Trials are currently being conducted to improve the quality of the image, treating the cameras with Rain Repellent (Turtle Wax), which has yielded positive results (Danish Agrifish Agency - unpublished data).

\subsection{Conclusion}

Despite technical and protocol challenges, REM has the potential to streamline the on-board monitoring of catches and discards in fishing fleet, as REM can be supplied with automatic counting, measuring and species identification if sufficient effort is put into development (French et al., 2015). The ultimate hurdle remains the reluctance of fishers to accept cameras on-board (Mangi et al., 2015; Plet-Hansen et al., 2016), along with political reluctance to enforce strict control in the fisheries.

\section{Acknowledgments}

The MINIDISC trial has been funded by the Ministry of Environment and Food of Denmark, under the European Fisheries Fund. Additional data analyses were performed with funding from the European Union's Seventh Framework Programme (FP7/20072013) under grant agreement MYFISH no 289257 and the European Union's Horizon 2020 research and innovation program under Grant Agreement DiscardLess No 633680. We also greatly appreciate the assistance given from Henrik Lund and the Danish Fisheries and Producers Organization (DFPO) in establishing contact and communication with the fishers. Lastly, a big thanks to the participating fishers and video inspectors, who made it possible.

\section{References}

Ames, R.T., Leaman, B.M., Ames, K.L., 2007. Evaluation of video technology for monitoring of multispecies longline catches. N. Am. J. Fish. Manag. 27, 955-964, http://dx.doi.org/10.1577/M06-029.1.

Dalskov, J., Kindt-Larsen, L., 2009. Final report on fully documented fishery. DTU Aqua Rep., http://dx.doi.org/10.1002/igs.1008, no. 204, pp 49.

EU, 2013. REGULATION (EU) No 227/2013 OF THE EUROPEAN PARLIAMENT AND OF THE COUNCIL of 13 March 2013 amending Council Regulation (EC) No $850 / 98$ for the conservation of fishery resources through technical measures for the protection of juveniles of marine organisms, Official Journal of the European Union.

French, G., Fisher, M.H., Mackiewicz, M., Needle, C.L., 2015. Convolutional neural networks for counting fish in fisheries surveillance video. In: Amaral, T., Matthews, S., Plötz, T., McKenna, S., Fisher, R. (Eds.), Proceedings of the Machine Vision of Animals and Their Behaviour (MVAB). , p. 7. 1-7.10.

Kindt-Larsen, L., Kirkegaard, E., Dalskov, J., 2011. Fully documented fishery: a tool to support a catch quota management system. ICES J. Mar. Sci. 68, 1606-1610, http://dx.doi.org/10.1093/icesjms/fsr065.

Mangi, S.C., Dolder, P.J., Catchpole, T.L., Rodmell, D., de Rozarieux, N., 2015. Approaches to fully documented fisheries: practical issues and stakeholder perceptions. Fish Fish. 16, 426-452, http://dx.doi.org/10.1111/faf.12065.

2013. Under $10 \mathrm{~m}$ Remote Electronic Monitoring Technical Trial.

McElderry, H., Schrader, J., Illingworth, J., 2003. The Efficacy of Video-Based Monitoring for the Halibut Longline. Fisheries and Oceans Canada, Victoria, Canada, ISSN 1480-4883.

Mortensen, L.O., Ulrich, C., Eliasen, S.Q., Olesen, H.J., 2016. Reducing discards without reducing profit: free gear choice in a Danish result-based management trial. ICES J. Mar. Sci. (in press).

Needle, C.L., Dinsdale, R., Buch, T.B., Catarino, R.M.D., Drewery, J., Butler, N., 2015. Scottish science applications of remote electronic monitoring. ICES J. Mar. Sci. 72, 1214-1229, http://dx.doi.org/10.1093/icesjms/fsu225.

Plet-Hansen, K.S., Mortensen, L.O., Olesen, H.J., Bergsson, H., Qvist Eliasen, S., Ulrich, C., 2016. Remote electronic monitoring and the landing obligation-some insights into fishers' and fishery inspectors' opinions. Mar. Policy (in press).

R Core Team, 2014. R: A Language and Environment for Statistical Computing.

Stanley, R., Olsen, N., Fedoruk, A., 2009. Independent validation of the accuracy of Yelloweye Rockfish catch estimates from the Canadian groundfish integration pilot project. Mar. Coast. Fish. Dyn. Manag. Ecosyst. Sci. 1, 354-362, http://dx. doi.org/10.1577/C09-005.1.

Stanley, R.D., McElderry, H.I., Mawani, T., Koolman, J., 2011. The advantages of an audit over a census approach to the review of video imagery in fishery monitoring. ICES J. Mar. Sci. 68, 1621-1627, http://dx.doi.org/10.1093/icesjms/ fsr058.

Ulrich, C., Olesen, H.J., Bergsson, H., Egekvist, J., Hakansson, K.B., Dalskov, J., Kindt-Larsen, L., Storr-Paulsen, M., 2015. Discarding of cod in the Danish fully documented fisheries trials. ICES J. Mar. Sci. 72, 1848-1860, http://dx.doi.org/ 10.1093/icesjms/fsv028.

van Helmond, A.T.M., Chen, C., Poos, J.J., 2015. How effective is electronic monitoring in mixed bottom-trawl fisherie? ICES J. Mar. Sci. 72, 1192-1200, http://dx.doi.org/10.1093/icesjms/fst048. 